\title{
Progress of Single Flux Quantum Packet Switch Technology
}

\author{
S. Yorozu, Y. Kameda, Y. Hashimoto, H. Terai, A. Fujimaki, and N. Yoshikawa
}

\begin{abstract}
With regard to sustaining future capacity of network traffic, the current hardware technology has a big bottleneck in terms of power consumption and insufficient operation speed. To overcome this bottleneck, we have developed a single flux quantum (SFQ) packet-switching circuit. We report recent progress in developing the SFQ packet-switching application. First, we demonstrate a $40 \mathrm{GHz}, 160 \mathrm{~Gb} / \mathrm{s}, 4 \times 4$ packet switching circuit, which is currently the fastest clock frequency reported packet switch. Then, we describe a currently possible switching module using SFQ technology. Finally, we consider how SFQ technology can be used to scale the packet-switching capacity up to 100 Tbps.
\end{abstract}

Index Terms-Multichip module, packet switching, single flux quantum circuit.

\section{INTRODUCTION}

W ITH recent progress in the Internet and other networks, the routing capacity per unit volume has doubled every 18 months. The power consumption of routers increases with their capacity, so it is now difficult to package a router in one rack of equipment. About $10 \mathrm{~kW}$ of power dissipation per rack is the limit of operation. As long as we only use current semiconductor technology, this limit corresponds to a switching capacity of approximately several $\mathrm{Tb} / \mathrm{s}$ for one rack. If Internet traffic continues to double every year, in nine years the traffic volume will be eight times greater than the capacity of the individual routers. Therefore it is difficult to sustain future volumes of network traffic.

Single flux quantum (SFQ) superconducting technology can overcome such constraints, (see Hayakawa [1] and references therein). Currently, a typical SFQ-based logic circuit such as an RS flip-flop circuit can achieve a clock speed of over $50 \mathrm{GHz}$ with only $10 \mu \mathrm{W}$ of power consumption. The large-scale circuit integration availability in this frequency domain is better than that with semiconductor technologies. By applying this circuit technology, the switching fabric could route packets without complex, massive parallelization. In this paper, we describe a prototype $40 \mathrm{GHz} 4 \times 4$ switching circuit, which is the fastest packet switch yet reported (a preliminary report is given in [2]).

Manuscript received October 4, 2004. This work was supported by the New Energy and Industrial Technology Development Organization (NEDO) as a Superconductor Network Device Project.

S. Yorozu, Y. Kameda, and Y. Hashimoto are with the Superconductivity Research Laboratory, ISTEC, Tsukuba, Ibaraki 305-8501, Japan (e-mail: yorozu@istec.or.jp; kameda@istec.or.jp; hasimoto@istec.or.jp).

H. Terai is with the National Institute of Information and Communications Technology (NICT), Kobe, Japan (e-mail: terai@nict.go.jp).

A. Fujimaki is with Nagoya University, Nagoya, Japan (e-mail: fujimaki@ nuee.nagoya-u.ac.jp).

N. Yoshikawa is with Yokohama National University, Yokohama, Japan (e-mail: yoshi@yoshilab.dnj.ynu.ac.jp).

Digital Object Identifier 10.1109/TASC.2005.849862
And we also show how to implement a large-scale switching capacity by using SFQ technology.

\section{SFQ PACKET SwITCH}

\section{A. Demonstration of a $4 \times 4$ Switch}

As a first prototype, we designed and tested a $4 \times 4$ packet switching circuit, which consists of four $2 \times 2$ switches. In the circuit design stage, we implemented a cell place and route $(\mathrm{P} \& \mathrm{R})$ tool and interconnection technology in order to establish design scalability for the circuit. The P\&R tool can automatically solve the problem of physical circuit layout by using concurrent-flow clocking and maintaining picosecond-order timing constraints, and it is the first automatic cell $\mathrm{P} \& \mathrm{R}$ tool for SFQ circuits [3]. There is no upper theoretical limit on the designable circuit scale with this tool. This enables us to design larger, more complex SFQ circuits than ever before. We generated the physical layout of the $2 \times 2$ switch by using the $\mathrm{P} \& \mathrm{R}$ tool. The resulting $2 \times 2$ circuit had 577 Josephson junctions. We confirmed experimentally that it worked for clock speeds up to $40 \mathrm{GHz}$. For interconnection between logic gates, a traditional SFQ circuit uses Josephson transmission lines (JTL's), so the latency of such a circuit tends to become large, which is a serious problem for some digital applications. Thus, the interconnection method is a key technology for increasing circuit performance. Replacing JTL interconnections with passive transmission lines (PTL) is very effective for further decreasing the circuit latency. We have recently improved the design methodology so as to reduce the impedance mismatch problem between PTL's and circuits [4], and we applied this approach in the $4 \times 4$ circuit design.

Fig. 1 shows a photograph of the $4 \times 4$ crossbar switch circuit. Six PTL's were used for connecting the four $2 \times 2$ switches. To satisfy the timing constraints for $40 \mathrm{GHz}$ clock operation, we equalized the lengths of the PTL's as much as possible. By applying the PTL's, we decreased the latency of the circuit block interconnection from $170 \mathrm{ps}$ to $24 \mathrm{ps}$. A circuit was then fabricated by using NEC $2 \mu \mathrm{m}$ square Nb Josephson junction technology, where $\mathrm{Jc}=2.5 \mathrm{kA} / \mathrm{cm}^{2}$ [5]. This circuit consisted of one Josephson junction layer, three $\mathrm{Nb}$ superconductor layers, and one Mo metal layer for the resistor. The minimum line and space was $1.5 \mu \mathrm{m}$. The power consumption of the $4 \times 4$ circuit was $0.8 \mathrm{~mW}$. The whole circuit size was $2.8 \mathrm{~mm} \times 3.4 \mathrm{~mm}$, with 4316 Josephson junctions. The circuit was tested in liquid helium at $4.2 \mathrm{~K}$ with an on-chip test circuit, which is a common way of testing SFQ circuits [6]. We confirmed the correct operation of all possible functions up to $40 \mathrm{GHz}$. Fig. 2 shows the frequency dependence of the measured bias current margin. 


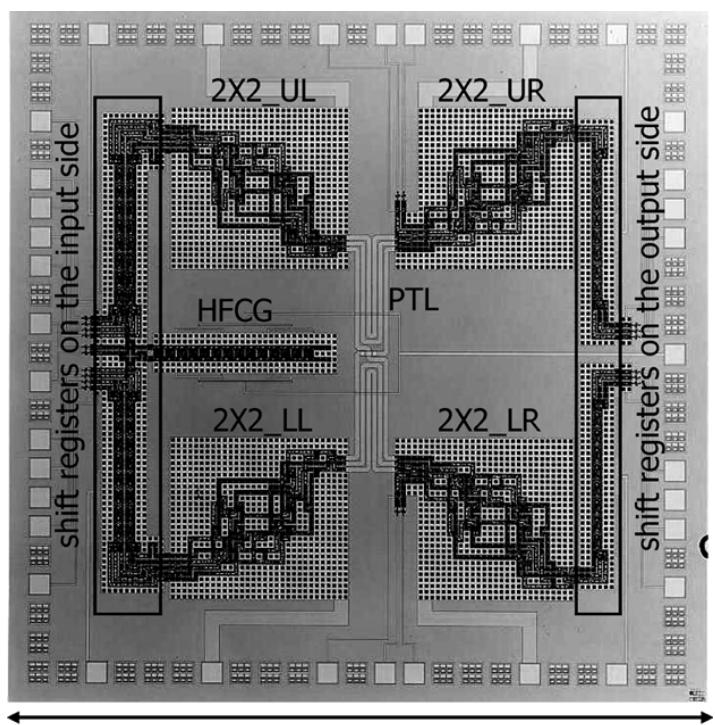

$5 \mathrm{~mm}$

Fig. 1. Die photograph of the $4 \times 4$ crossbar switch.

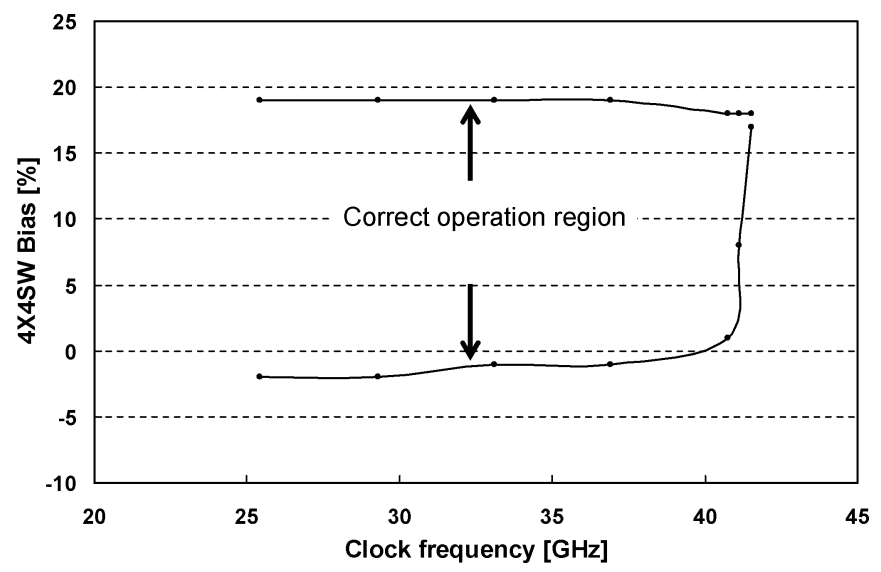

Fig. 2. Measured bias margin from on-chip testing.

\section{B. Packet-Switching Subsystem Packaging}

As compared with semiconductor technology, an SFQ switching fabric can process packets without parallelization, even though the packet speed can reach $40 \mathrm{~Gb} / \mathrm{s}$. Thus, smaller, simpler packet switches can be constructed, as compared to conventional ones. The performance as a subsystem, however, strongly depends on the packaging technology. Fig. 3 shows one possible schematic for the superconducting subsystem. We assume that the subsystem is mounted on a two-stage cryo-cooler [7]. The package has two separate temperature stages, operating at $5 \mathrm{~K}$ and around $40 \mathrm{~K}$. In the $40 \mathrm{~K}$ stage, the interconnection cables are thermally anchored. On the output side, output signals are amplified by two kinds of amplifier. We have developed a two-stage signal amplification scheme. In the first stage, an output signal is first amplified to several milivolts by using a superconducting digital voltage driver, which is based on Josephson junction latching type driver [8]. It is then amplified again to several hundred milivolts by using a semiconductor amplifier operating in the $40 \mathrm{~K}$ stage. We have developed a low-temperature semiconductor amplifier which is based on GaAs FET technology, and confirmed its low-BER

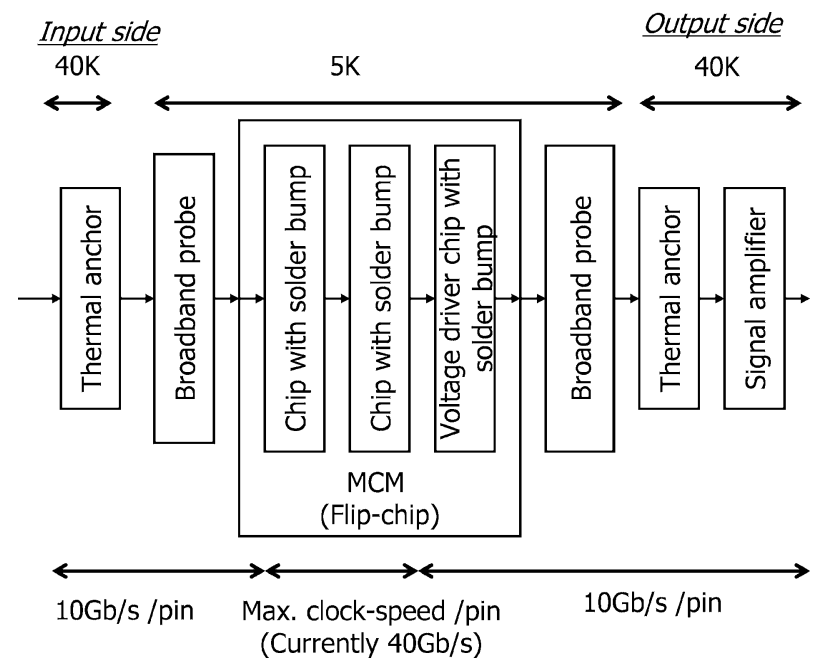

Fig. 3. A possible block diagram of SFQ subsystem.

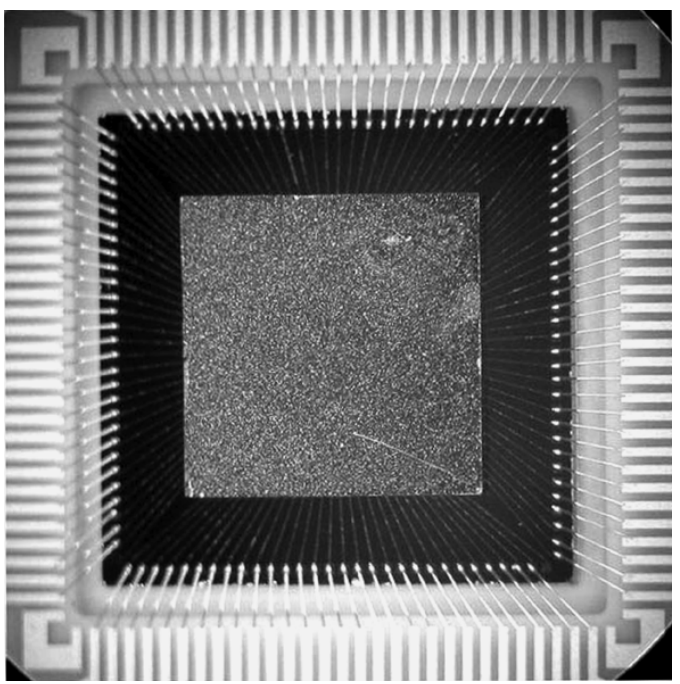

Fig. 4. Photograph of a flip-chip example.

amplification $(20 \mathrm{~dB})$ operation for a $2 \mathrm{mV}, 12 \mathrm{~Gb} / \mathrm{s}$ input signal at $40 \mathrm{~K}$.

Multi-chip-module (MCM) technology is an important technology for SFQ circuits. We have developed a flip-chip connection technology using superconducting solder bumps. Pads are deposited on both the MCM substrate and the chip by sputtering $\mathrm{Ti} / \mathrm{Pd} / \mathrm{Au}$ on $\mathrm{Nb}$. The solder bumps are then formed on both the MCM substrate and the chip by immersing it [9]. The solder material is a $52 \%-\mathrm{In} / 48 \%$-Sn alloy with a melting point of $118^{\circ} \mathrm{C}$ [10]. The heights of the bumps are approximately $5 \mu \mathrm{m}$, on 50 $\mu \mathrm{m}$-diameter pads. The flip-chip attachment of the chip to the substrate is done by using a flip-chip bonder. The XY alignment resolution of the bonder is $3 \mu \mathrm{m}$. Fig. 4 shows an example of a flip-chip test sample. The size of the substrate is $8 \mathrm{~mm} \times 8 \mathrm{~mm}$, and the size of the chip is $5 \mathrm{~mm} \times 5 \mathrm{~mm}$. The diameter of the bump is $50 \mu \mathrm{m}$. The substrate is wire-bonded to a ceramic carrier. On the MCM, chip-to-chip communication is done with an SFQ pulse, except for any speed degradation with the specially designed driver and receiver pair.

The target off-MCM interconnection speed between the MCM and a room-temperature region is $10 \mathrm{~Gb} / \mathrm{s}$ per pin. 


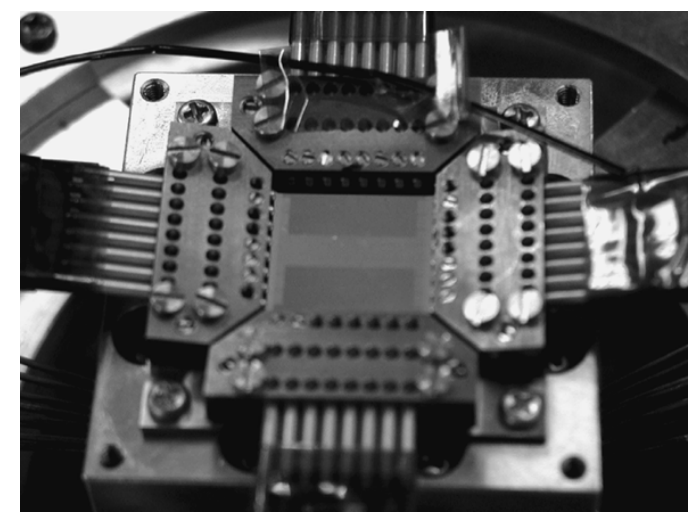

Fig. 5. Photograph of a packaging example for probe pins.

For high-end digital applications, a multiple-pin, wide-band probing technology is needed, which is a very challenging problem. Currently, we have developed a prototype wide-band probe package consisting of 32 pins. Each pin is designed for DC to $60 \mathrm{GHz}$ bandwidth, co-planar-type contact between the MCM and an interconnection cable. Fig. 5 shows a first-prototype implementation of this package.

\section{SWITCHING CAPACITY SCALABILITY}

\section{A. Single-Rack, Centralized Approach for 10-Tb/s-Scale Throughput}

There are two ways to expand the switching capacity. One is to increase the capacities of the crossbar switch and the switch scheduler in a single-stage system. The second way to expand the switching capacity is to apply a multi-rack system with a distributed switch. Multi-rack routers spread the system power over multiple racks, thus it has an advantage of reducing the power density. However, multi-rack systems with distributed, multistage switching fabrics such as Benes, Banyan, Hypercube, etc., have insufficient performance to guarantee throughput. This stands in contrast with single-stage switches, for which throughput can be guaranteed. Therefore, it should be noted that the centralized approach is generally superior to the distributed approach for achieving more effective performance. Providing guaranteed throughput would normally require a complicated centralized scheduler to configure the switching fabric. The scheduler speed will grow slowly because of its $\mathrm{O}\left(\mathrm{N}^{2}\right)$ complexity. Even if the arbitration is distributed over multiple ASIC's, the interconnect power still scales with $\mathrm{O}\left(\mathrm{N}^{2}\right)$. One reported high-speed centralized scheduler controls 256 ports at 10 Gbps [11]. This design is limited by the power dissipation and pin counts of the scheduler ASIC's, which shows it is difficult to scale centralized schedulers by using conventional technology, with an increase in the number of ports, or in the line rate. Applying an SFQ switch scheduler, on the other hand, would enable greater expansion. An SFQ circuit is suitable for implementing a switch scheduler because of its high-speed clock operation without huge power consumption. We have developed an SFQ switch scheduler based on the iSLIP algorithms [12]. Fig. 6 shows a photograph of the fabricated prototype round-robin arbiter for the scheduler. The arbiter scans all input port requests for one output port,

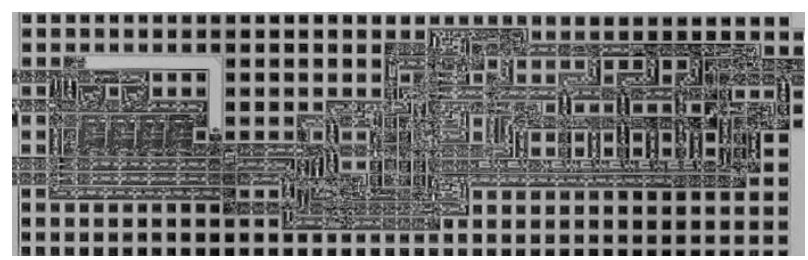

Fig. 6. Photograph of the fabricated prototype switch scheduler.

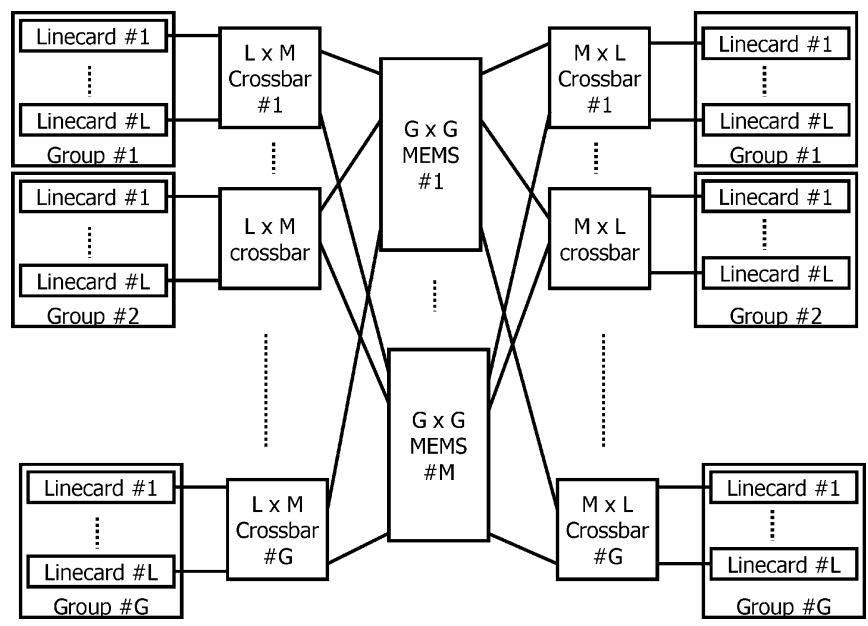

Fig. 7. Block diagram of a load-balanced architecture.

then arbitrates all and gives grant signals to selected ports. The scanning has to be serial for all ports. Thus, the performance of the scheduler depends on the scanning operational clock cycle. We have already confirmed this circuit's operation at $40 \mathrm{GHz}$, so it is very promising for providing higher throughput [13].

\section{B. Toward Throughput of Over 100 Tbps}

Recently, the load-balanced switch architecture has been proposed for large-scale throughput switching [14]. The main advantage of the load-balanced switch is that it works without a centralized scheduler, so there is no degradation of throughput guarantees by the scheduler. Fig. 7 shows an example of a block diagram of this architecture [15]. It consists of $(\mathrm{L} \times \mathrm{G})$ linecards for packet processing, $(\mathrm{G} \times 2)$ crossbar switches, and $\mathrm{M}$ optical MEMS's for forwarding packets. The MEMS switches are statistically interconnected crossbar switches. The combination of crossbar switches and MEMS realizes load balancing of incoming packets and as a result, there are no packet conflicts in the switch. The most challenging part of the system is to build the electronic crossbar switches connecting the linecards. From architectural considerations [15], a several Tb/s crossbar switching capacity is required to achieve greater than a $100 \mathrm{~Tb} / \mathrm{s}$ throughput. The capacity might exceed the upper limit of a crossbar chip implementation capability using semiconductor circuits. Thus it is difficult to increase the total throughput over $100 \mathrm{~Tb} / \mathrm{s}$. In contrast, by using SFQ circuit technology, we might be able to actually develop such a Tb/s-scale switch module. Therefore, we propose to use SFQ crossbar switches in the load-balanced architecture to develop a router system operating at over $100 \mathrm{~Tb} / \mathrm{s}$. If we adopt the load-balanced architecture, the heat load due to interconnection between the 
room-temperature and low-temperature regions can be reduced, because the number of interconnection cables would be reduced as compared with a $100 \mathrm{~Tb} / \mathrm{s}$ single-rack-type router. In this case, major issue is how to implement SFQ/optical interface. SFQ/electrical/optical interface by using conventional laser diode at room temperature is a currently possible solution, but we should consider further way such as SFQ/optical direct interfacing.

\section{CONCLUSION}

We have described the recent progress in SFQ-circuit-based packet switching and its associated module technology. For circuit operation, we have demonstrated a $4 \times 4,160 \mathrm{~Gb} / \mathrm{s}$ packet-switching circuit. Such a circuit could be packaged by applying technology based especially on novel multi-chip module (MCM) and probing technology development. In addition, we have considered a possible approach for achieving switching capacity scalability up to $100 \mathrm{~Tb} / \mathrm{s}$. Up to the level of several tens of terabits per second, the combination of an SFQ-based centralized packet switch and a switch scheduler is sufficient. To achieve $100 \mathrm{~Tb} / \mathrm{s}$, a load-balanced implementation with SFQ crossbar switches is a promising approach. In any event, SFQ technology will clearly play an important role in high-end switching for communication networks.

\section{ACKNOWLEDGMENT}

The authors would like to thank Dr. Hinode, Ms. Isaka, Mr. Kamei, Mr. Kitagawa, Dr. Nagasawa, Dr. Hidaka, and Prof. Hayakawa for their help with the circuit fabrication technology. They also thank Dr. Keslassy and Dr. Harai for discussing packet-switching architectural issues.

\section{REFERENCES}

[1] H. Hayakawa, N. Yoshikawa, S. Yorozu, and A. Fujimaki, "Superconducting digital electronics," Proc. IEEE, vol. 92, no. 10, pp. 1549-1563, Oct. 2004.

[2] S. Yorozu, Y. Hashimoto, Y. Kameda, H. Terai, A. Fujimaki, and N. Yoshikawa, "A $40 \mathrm{GHz}$ clock $160 \mathrm{~Gb} / \mathrm{s} 4 \times 4$ switch circuit using single flux quantum technology for high-speed packet switching systems," in Proc. IEEE 2004 Workshop High Performance Switching and Routing, 2004, pp. 20-23.

[3] Y. Kameda, S. Yorozu, H. Terai, and A. Fujimaki, "High-speed operation of a single-flux-quantum (SFQ) cross/bar switch up to $35 \mathrm{GHz}$," Jpn. J. Appl. Phys., vol. 42, pp. 2163-2166, 2003.

[4] Y. Hashimoto, S. Yorozu, Y. Kameda, and V. Semenov, "A design approach to passive interconnects for single flux quantum logic circuits," IEEE Trans. Appl. Supercond., vol. 13, pp. 535-538, 2003.

[5] S. Nagasawa, Y. Hashimoto, H. Numata, and S. Tahara, "A 380 ps, 9.5 mW Josephson 4-Kbit RAM operated at a high bit yield," IEEE Trans. Appl. Supercond., vol. 5, pp. 2447-2452, Jun. 1995.

[6] A. Kirichenko, O. Mukhanov, and A. Ryzhikh, "Advanced on-chip test technology for RSFQ circuits," IEEE Trans. Appl. Supercond., vol. 7, pp. 3438-3441, 1997.

[7] D. Gupta, A. Kadin, R. Webber, I. Rochwarger, D. Bryce, W. Hollander, Y. Yim, Y. Channakeshav, R. Kraft, J.-W. Kim, and J. McDonald, "Integration of cryocooled superconducting analog-to-digital converter and SiGe output amplifier," IEEE Trans. Appl. Supercond., vol. 13, pp. 477-483, 2003.

[8] N. Harada, N. Yoshikawa, K. Yoda, A. Yoshida, and N. Yokoyama, "Logic operation at $5 \mathrm{Gbps}$ of an output interface for single flux quantum systems," IEEE Trans. Appl. Supercond., vol. 13, no. 3, pp. 3814-3816, 2003.

[9] R. Sandell, G. Akerling, and A. Smith, "Multi-chip packaging for high speed superconducting circuits," IEEE Trans. Appl. Supercond., vol. 5, pp. 3160-3163, 1995.

[10] M. Hansen and K. Anderko, Constitution of Binary Alloys: McGrawHill, 1998.

[11] N. McKeown, C. Calamvokis, and S.-T. Chuang, "A 2.5 Tb/s LCS switch core," in Proc. Hot Chips XIII, Aug. 2001.

[12] N. McKeown, "The iSLIP scheduling algorithm for input-queued switches," IEEE/ACM Trans. Netw., vol. 7, no. 42, pp. 188-201, Apr. 1999.

[13] Y. Kameda, S. Yorozu, Y. Hashimoto, H. Terai, A. Fujimaki, and N. Yoshikawa, "Single-flux-quantum (SFQ) circuit design and test of crossbar switch scheduler," in Proc. 2004 Appl. Supercond. Conf., to appear in IEEE Trans. Appl. Supercond., 2005.

[14] C. S. Chang, D. S. Lee, and Y. S. Jou, "Load balanced Birkhoff-von Neumann switches, part I: one-stage buffering," in Proc. IEEE HPSR '01, Dallas, May 2001.

[15] I. Keslassy, S.-T. Chuang, K. Yu, D. Miller, M. Horowitz, O. Solgaard, and N. McKeown, "Scaling internet routers using optics," in Proc. ACM SIGCOMM '03, Karlsruhe, Germany, Aug. 2003. 